\title{
Energy Resolved Imaging using the GP2 Detector: Progress in Instrumentation, Methods and Data Analysis
}

\author{
Daniel E. Pooley ${ }^{1, a^{*}}$, Jason W. L. Lee ${ }^{2, b}$, Freddie A. Akeroyd ${ }^{1, c}$, Owen Arnold ${ }^{1, d}$, \\ Michael Hart ${ }^{1, \mathrm{e}}$, Jaya J. John ${ }^{4, f}$, Peter M. Kadletz ${ }^{3, \mathrm{~g}}$, Winfried Kockelmann ${ }^{1, \mathrm{~h}}$, \\ Triestino Minniti $^{1, i}$, Christopher Moreton-Smith ${ }^{1, j}$, Manuel Morgano ${ }^{5, k}$, \\ Nigel J. Rhodes ${ }^{1, I}$, Erik M. Schooneveld ${ }^{1, m}$, Iain Sedgwick ${ }^{1, n}$, Claire Vallance ${ }^{2,0}$, \\ Robin Woracek ${ }^{3, p}$ \\ ${ }^{1}$ STFC, Rutherford Appleton Laboratory, Harwell Campus, Didcot OX11 0QX UK \\ ${ }^{2}$ Department of Chemistry, Chemistry Research Laboratory, 12 Mansfield Road, University of \\ Oxford, Oxford, OX1 3TA UK \\ ${ }^{3}$ European Spallation Source ERIC, SE-221 00, Lund, Sweden \\ ${ }^{4}$ Department of Physics, Denys Wilkinson Building, Keble Road, University of Oxford, \\ Oxford OX1 3RH UK

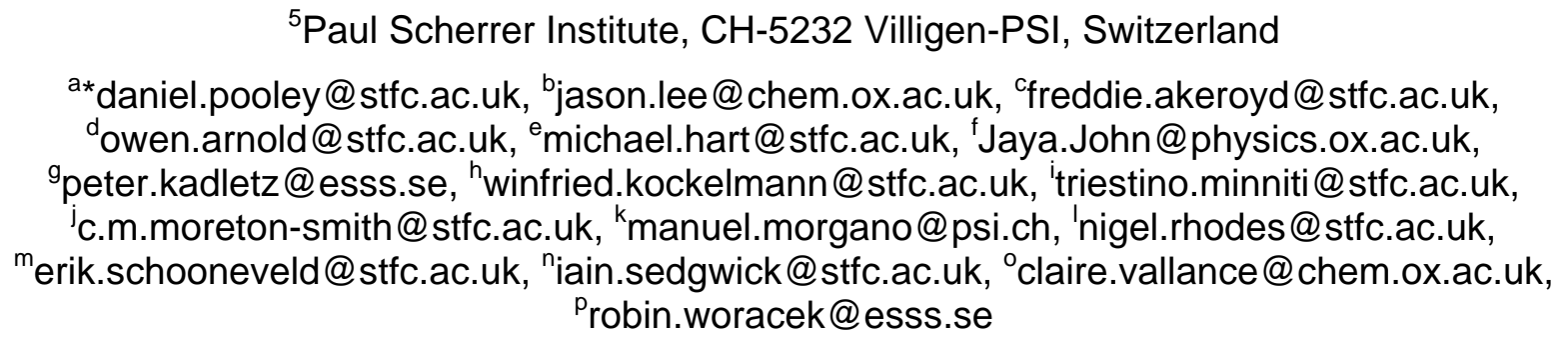

Keywords: Neutron, Energy Resolved, Wavelength Dispersive, Imaging, Tomography, CMOS, Gadolinium, Pixel, Spallation, ToF, Time of Flight

\begin{abstract}
We report on the recent developments of the 'GP2' detector, highlighting a selection of energy resolved measurements and associated methodology. GP2 is a 100k pixel time-offlight (ToF) neutron camera, which combines a gadolinium converter film and a CMOS (Complementary Metal Oxide Semiconductor) readout sensor. This paper describes an up-todate specification of the detector and its variants, progress that has been made towards integration into the Imaging and Materials Science instrument (IMAT) and an independent review at the ESS test beamline, V20. Two ToF data reduction methods are detailed, namely wavelength dispersive contrast enhancement and 'wavelength frame multiplication' (WFM) reduction.
\end{abstract}

\section{Introduction to GP2}

The 'GP' detector series has been developed over the last six years as a collaborative project between STFC Technology Department, Oxford University and the ISIS Neutron and Muon source, all based in Oxfordshire, UK. The acronym GP derives from the use of gadolinium as a neutron converter and the implementation of an event-mode sensor developed for applications in imaging mass spectrometry, known as the 'PImMS' sensor [1]. GP1 was a scaled-down prototype detector with an active area of $\approx 25 \mathrm{~mm}^{2}$. The second generation (GP2) detector shown in Fig 1 has a greatly increased area, $22.7 \times 22.7 \mathrm{~mm}^{2}$. Variants currently in development include the F-mode, where a full sensor readout is reduced to $\sim 3 \mathrm{~ms}$, the S-mode, where the sensor is sandwiched between two layers of gadolinium to enhance neutron detection efficiency and $\mathrm{M}$, 
where the sensor is implemented in modular form to facilitate sparse tiling over large areas. A concise, tabulated, specification of GP2 is given in Table 1, with full detail reported in [2]. The 'register' feature is sensor-level memory allowing up to 4 events per pixel to be buffered between readout operations. This paper focuses on the recent use of the detector at two time-offlight imaging beamlines: IMAT [3] at the ISIS source, UK, and the ESS test beamline V20 [4] located at HZB, Germany. In the case of IMAT, where the detector is available to the user programme, further detail is provided to describe integration into the existing beamline infrastructure.

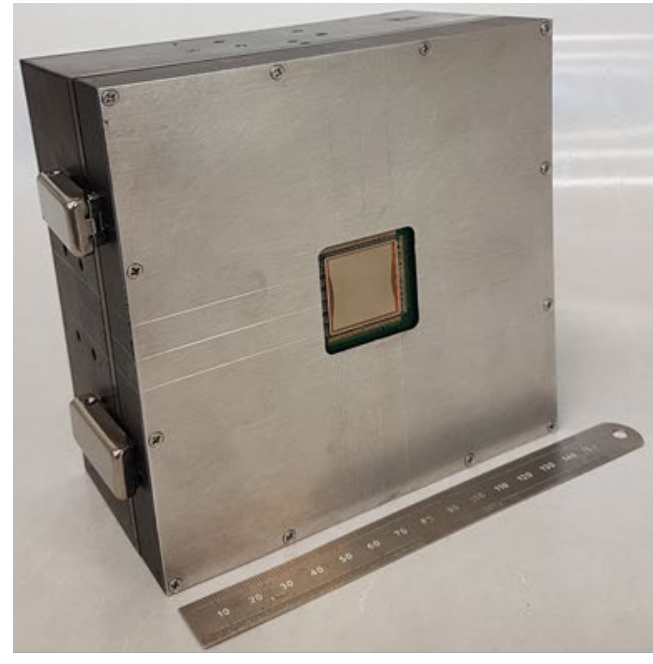

Fig 1. GP2 detector with protective cover removed to expose the $22.7 \times 22.7$ $\mathrm{mm}^{2}$ sensor.

\section{Integration into IMAT}

This section details the process of integrating GP2 into the IMAT infrastructure along with example IMAT data and ensuing contrast enhancement.

\subsection{Mechanical Integration.}

IMAT can be tailored to each measurement by selection of various instrument parameters, one of which is the camera system. Changes between different imaging detectors (cameras) are performed using a robotic arm, facilitating quick and accurate changes between white-beam imaging (time integrated) and time-of-flight (energy resolved) imaging. Each detector has its own plate, which provides a standardised interface (mating socket) for the robotic arm to locate and pickup. The plate for GP2 is shown below in Fig 2.
Table 1. Specification of the GP2 detector.

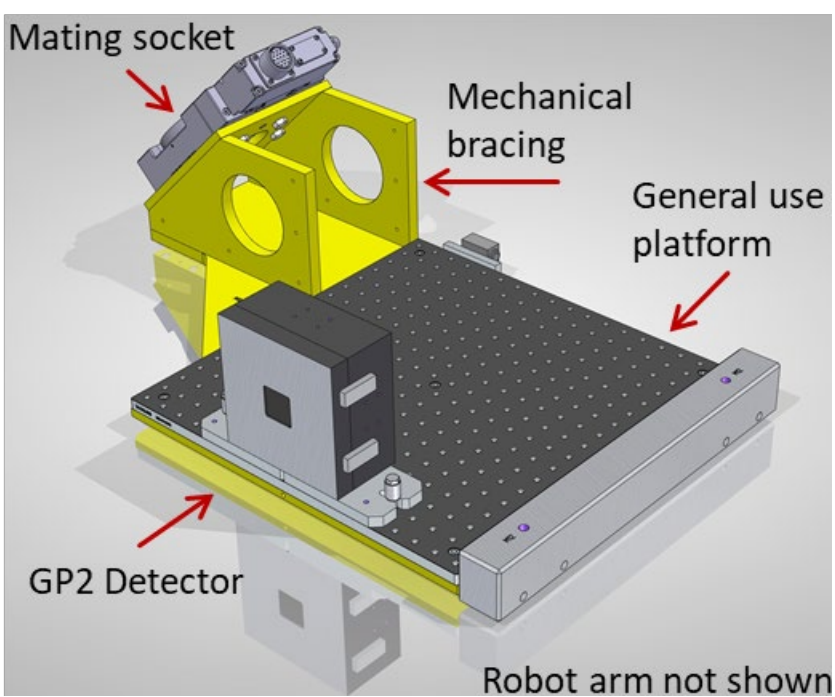

Fig 2. The robotic arm mounting plate for GP2. 


\subsection{Control and Data}

For GP2 to be available within the IMAT user programme the detector needed to be integrated into the ISIS EPICS [5] based control system. Key objectives associated with this were:

- Synchronisation of Start/Stop/Pause commands between the local GP2 acquisition computer and the IMAT instrument control computer.

- Passing file name constructors and meta-data between the local GP2 acquisition computer and the IMAT instrument control computer.

- Stream data live to user and ISIS archive.

As the camera software is written in LabVIEW, a convenient control mechanism was network shared variables [6], for which generic EPICS support had previously been developed [7]. These network shared variables allow setting and reading of values remotely from the instrument control computer and hence scripting of the camera operation via EPICS using Python.

The data is currently saved as a binary file containing three unsigned 16 bit integers for each hit. Each frame is delimited by two 32 bit integers, providing the array size. The longer-term aim is to store the data in a HDF5 format, namely NeXus [8], which will ensure compatibility with the ISIS data archive and reconstruction software such as SAVU [9].

\subsection{IMAT Data: 4 Registers.}

To illustrate the 4-memory register capability of GP2 and to enumerate the typical count rate on IMAT, open beam data with a $40 \mathrm{~mm}$ pinhole (L/D 250) was recorded, with GP2 synchronous to the $10 \mathrm{~Hz}$ source. In this configuration GP2 measured $\sim 1.1 \times 10^{5}$ hits per $100 \mathrm{~ms}$ frame, more than the total number of pixels $(324 \times 324)$. Fig 3 shows the hits in each register as a histogram. The number of hits in each register decreases with increasing register number since the registers are populated sequentially starting at register 1 . Monitoring this distribution provides diagnostic information as to how close to saturation (missing counts) the detector is, mitigating the need for offline data correction. If the neutron flux were to increase, read-out within a frame would have to be performed at the cost of introducing time gaps in the data. In this example GP2 was operated as a 3-register camera as the fourth register was minimally occupied.

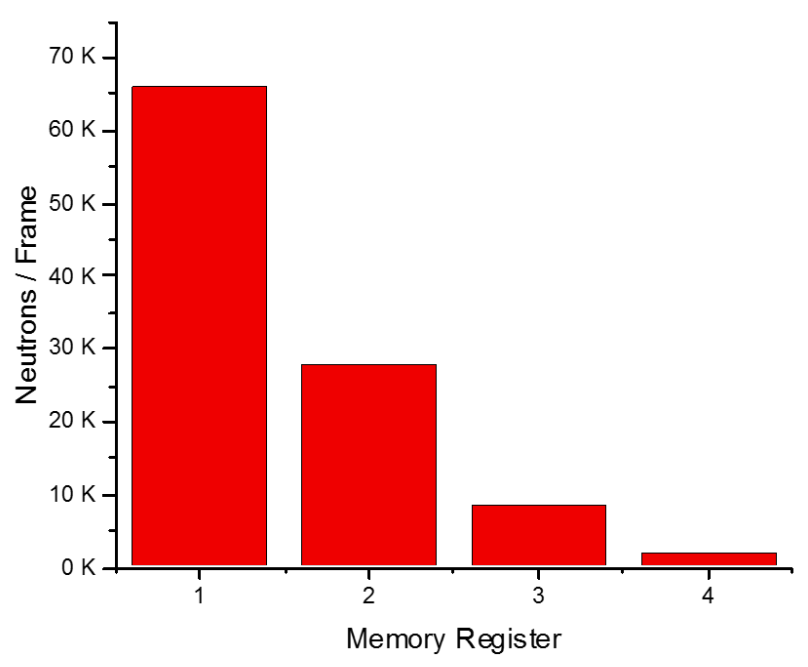

Fig 3. Histogram showing the hit-distribution across the 4 memory registers for a typical IMAT frame.

\subsection{IMAT Data: Contrast Enhancement}

GP2 is an event-mode detector meaning it records wavelength (energy) dispersive data. The benefits of using energy resolved neutron imaging to measure sample properties are widely reported [10] [11]. Here we emphasise that some simple diagnostic tools can be used to both ascertain the measurement quality and to provide contrast enhancement on-the-fly. A simple graphical user interface was developed which allows the user to maximise the grey-value contrast between two regions of interest (RoI) as shown in Fig 4. The program normalises the time-of-flight spectra for each RoI and displays the ratio (green). A simple thresholding of the 
ratio plot is used to select the time-of-flight bins (i.e. energy channels) which provide more contrast to the material in RoI-1, by applying this selection to the whole image.

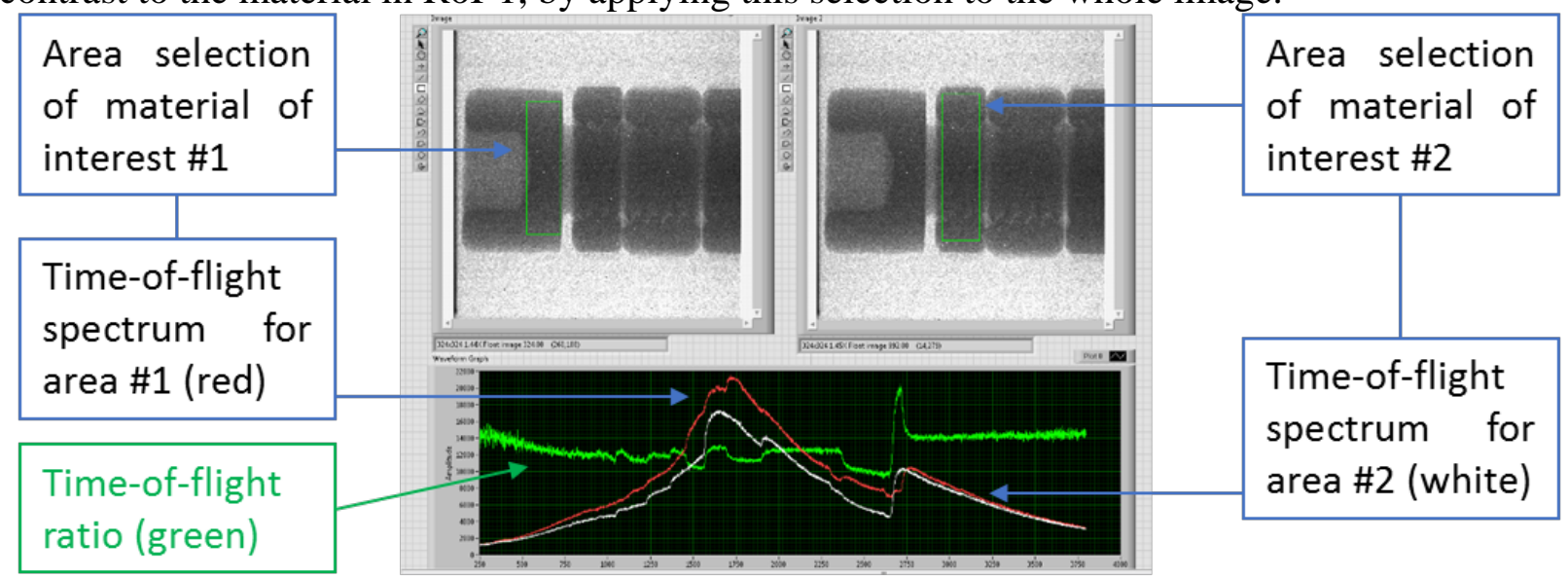

Fig 4. Screenshot of GUI used to select time channels to produce contrast enhanced image.

An example of this simple method is demonstrated using an energy resolved image of a bolt with alternating FCC and BCC steel nuts. Both materials have similar macroscopic cross sections and therefore attenuate the beam equally. In a whitebeam radiograph these two materials will have the same grey value and will be indistinguishable, as seen in Fig 4 top. However, by selecting all the ToF bins that enhance one of the materials those pixels can be transported to a different grey value. This method offers a simple 'online' analysis, which can immediately distinguish features without a large overhead in data analysis.
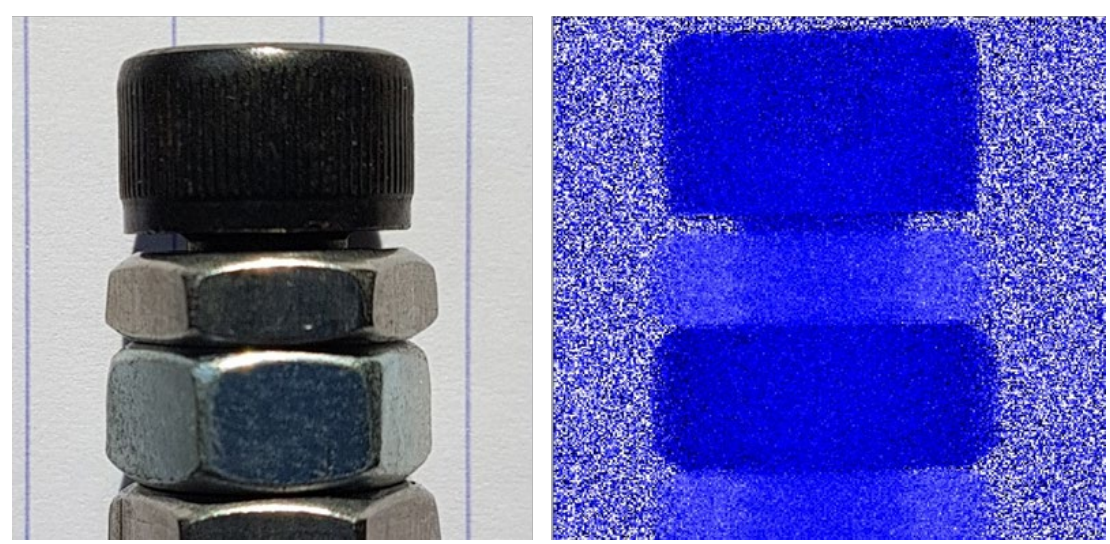

Fig 5. LEFT: Photo of sample, alternating BCC and FCC steel. RIGHT: Example output image, where the GUI has been used to enhance the FCC bolt and nut. Time channels were selected and integrated by thresholding the ratio plot (Fig 4 green).

\section{Independent Review: Measurements at the ESS Test Beamline, V20}

As the GP2 detector has reached a point of technological maturity, moving from the R\&D phase to deployment, impartial technology review from new users was sought. The aims of this exercise were threefold. Firstly, independent review is considered due diligence for any scientific development. Secondly new users to the technology will provide valuable feedback for future development. Thirdly, the suitability and compatibility with different source structures and flux distributions should be ascertained if GP2 is to be deployed to other facilities. To this end GP2 was tested at the dedicated ESS test beamline V20, located at the BER2 research reactor at the Helmholtz Zentrum Berlin. The beamline can mimic the time structure of the future European Spallation Source (ESS) using double-disk choppers and is equipped with optional pulse shaping choppers for 'Wavelength Frame Multiplication' (WFM). It is also dedicated to the testing and development of instrumentation, scientific methods and corresponding data reduction for the ESS [4] [12]. A number of measurements were performed, including count rate investigations, 
detector stability, source-structure compatibility and an in-situ tensile experiment. While the main detector parameters were found to be consistent with results previously reported and the latter experiment will be reported elsewhere in detail, we report here on two measurements particularly suited to the V20 beamline.

\subsection{Detector Stability}

Reactor based beamlines are inherently stable, providing an opportunity to investigate detector performance over hours and days. Fig 6 shows the variation between the integrated counts of an open-beam GP2 measurement (red) alongside the reactor power (blue) and cold source temperature (green). As the neutron flux is dependent on both the reactor power and source temperature, the observed correlation indicates that that detector stability is much better than the observed variation, i.e $<2 \%$. This type of analysis further demonstrates the advantages of both the detector and event mode data acquisition.

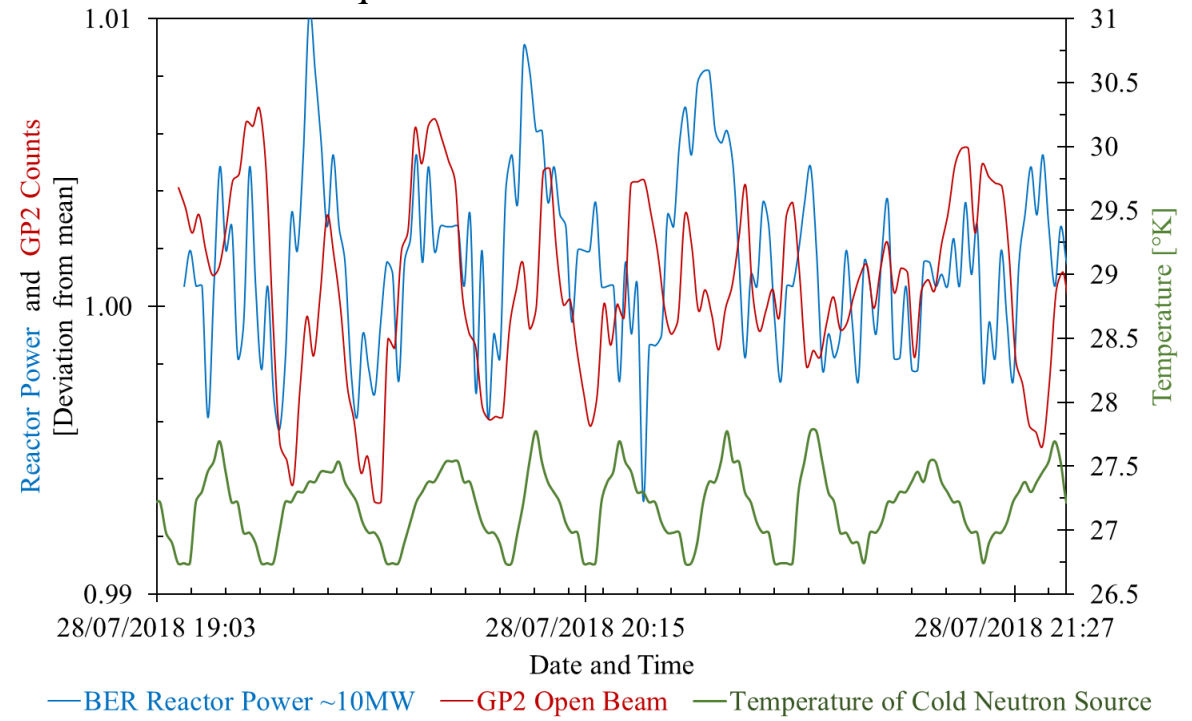

Fig 6. Stability plot showing fluctuation in reactor power (blue) and the counts measured with

GP2 (red) alongside the cold source temperature (green, right-axis) variation. The neutron counts measured with GP2 is sensitive to the compound effect of both reactor power and source temperature.

\subsection{Source Structure}

V20 mimics the ESS pulse structure: a 3 ms long pulse with a $14 \mathrm{~Hz}$ repetition rate. As at any ToF beamline, the wavelength resolution is determined by the length of the instrument, for the herein presented measurement between $\sim 5 \%$ (8 $\AA$ ) and $~ 20 \%$ (2 $\AA$ ) for a flight length of $\sim 26 \mathrm{~m}$. While beamlines at the ESS will typically be much longer, several of them will have additional pulse shaping choppers to improve the resolution, similar to V20. V20 is equipped with a chopper cascade to operate in wavelength frame multiplication (WFM) mode, described fully in [4]. In this mode, six new and much narrower source pulses are created from the long pulse, all with a constant and much higher resolution. These are separated in time but overlap in wavelength and hence form a continuous wavelength band after data reduction ('stitching' using the Mantid framework [13]). The resolution is furthermore tuneable by changing the distance between the WFM choppers and the herein utilised settings provide $\sim 2.3 \%$. This mode of operation offers a novel test for GP2, as the pulse structure requires a detector to handle large changes in flux with high stability. The measurement of a $10 \mathrm{~mm}$ thick BCC steel plate is shown in Fig 7, demonstrating both long pulse and WFM mode. By inspection of the Bragg edge features in the stitched data and observing its smooth continuous nature (Fig 7. RIGHT), the 
quality of GP2 data is evident. The WFM data shows sharper, well-resolved Bragg edges (black) suitable for advanced analysis methods not possible with the long pulse data. The stitching process applied to GP2 data and its application to more complex systems will be presented in a separate and more detailed publication.
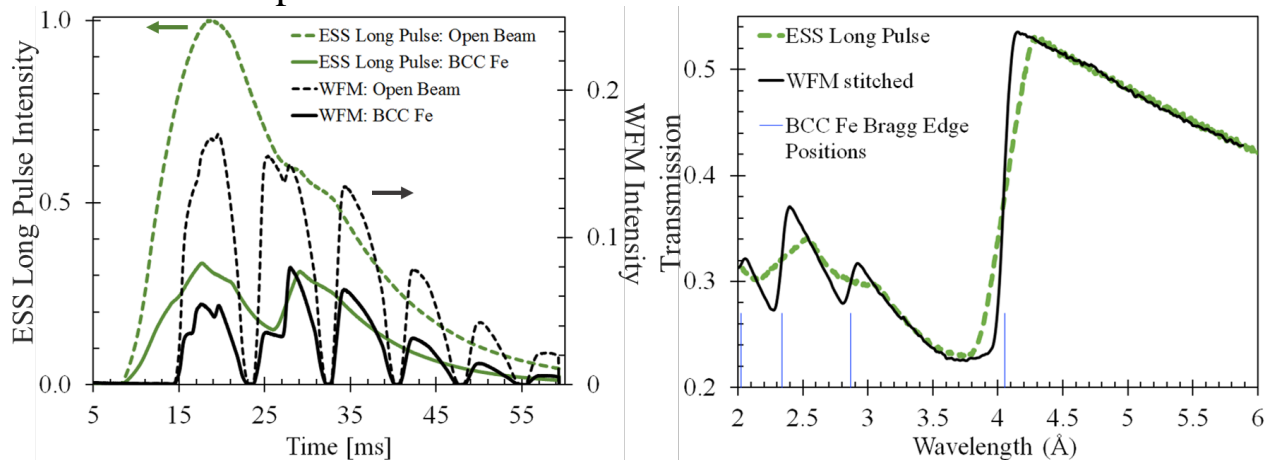

Fig 7. LEFT: Open beam and sample data, for both the 'ESS long pulse mode' and 'WFM mode'. Peak intensity of the long pulse mode data is normalised to unity (left y-axis). The same scale is applied to the WFM intensity (right y-axis). RIGHT: Transmission data, showing the difference in resolution between the two modes. The known BCC Bragg edge positions are indicated.

\section{Summary}

An overview of recent developments for GP2 has been presented, alongside two examples of ToF data reduction. With $\mathrm{F}, \mathrm{S}$ and $\mathrm{M}$ variants currently under development and the positive feedback from the imaging community we anticipate wider deployment across the facilities.

\section{References}

[1] I. Sedgwick, A. Clark, J. Crooks, R. Turchetta, L. Hill, J. J. John, A. Nomerotski, R. Pisarczyk, M. Brouard, S. H. Gardiner, E. Halford, J. W. Lee, M. L. Lipciuc, C. Slater, C. Vallance, E. S. Wilman, B. Winter and W. H. Yuen, "PImMS: A self-triggered, 25ns resolution monolithic CMOS sensor for Time-of-Flight and Imaging Mass Spectrometry," IEEE NEWCAS (2012). https://doi.org/10.1109/NEWCAS.2012.6329065

[2] D. E. Pooley, J. W. L. Lee, M. Brouard, J. J. John, W. Kockelmann, N. J. Rhodes, E. M. Schooneveld, I. Sedgwick, R. Turchetta and C. Vallance, "Development of the "GP2" Detector: Modification of the PImMS CMOS Sensor for Energy-Resolved Neutron Radiography," IEEE TNS, 64 (2017) 2979. https://doi.org/10.1109/TNS.2017.2772040

[3] T. Minniti, K. Watanabe, G. Burca and D. E. Pooley, "Characterization of the new neutron imaging and materials science facility IMAT," NIM A, 888 (2018) 184. https://doi.org/10.1016/j.nima.2018.01.037

[4] M. Strobl, M. Bulat and K. Habicht, "The wavelength frame multiplication chopper system for the ESS test beamline at the BER II reactor-A concept study of a fundamental ESS instrument principle.," NIM A, 705 (2013) 74. https://doi.org/10.1016/j.nima.2012.11.190

[5] [Online]. Available: https://epics-controls.org

[6] [Online]. Available: http://www.ni.com/white-paper/5484

[7] [Online]. Available: http://epics.isis.stfc.ac.uk/doxygen/NetShrVar

[8] M. Könnecke, F. A. Akeroyd, H. J. Bernstein, A. S. Brewster, S. I. Campbell, B. Clausen, S. Cottrell, J. U. Hoffmann, P. R. Jemian, D. Mannicke, R. Osborn, P. F. Peterson, T. Richter, J. 
Suzuki, B. Watts, E. Wintersberger and J. Wuttke, "The NeXus data format," J. Appl. Cryst,. 48 (2015) 301. https://doi.org/10.1107/S1600576714027575

[9] [Online]. Available: https://savu.readthedocs.io/en/latest/about

[10] R. Woracek, J. Santisteban, A. Fedrigo and M. Strobl, "Diffraction in neutron imaging-A review," NIM A, 878 (2018) 141. https://doi.org/10.1016/j.nima.2017.07.040

[11] N. Kardjilov, I. Manke, R. Woracek, A. Hilger and J. Banhart, "Advances in neutron imaging," Materials Today, 21 (2018) 652. https://doi.org/10.1016/j.mattod.2018.03.001

[12] R. Woracek, T. Hofmann, M. Bulat, M. Sales, K. Habicht, K. Andersen and M. Strobl, "The test beamline of the European Spallation Source - Instrumentation development and wavelength frame multiplication," NIM $\quad$ A, $\quad \mathbf{8 3 9} \quad$ (2016) 102. https://doi.org/10.1016/j.nima.2016.09.034

[13] O. Arnold, J.C. Bilheux, J.M. Borreguero, A. Buts, S.I. Campbell, L. Chapon, M. Doucet, N. Draper, R. Ferraz Leal, M.A. Gigg, V.E. Lynch, A. Markvardsen, D.J. Mikkelson, R.L. Mikkelson, R. Miller, K. Palmen, P. Parker, G. Passos, T.G. Perring, P.F. Peterson, S. Ren, M.A. Reuter, A.T. Savici, J.W. Taylor, R.J. Taylor, R. Tolchenov, W. Zhou, J. Zikovsky, "MantidData analysis and visualization package for neutron scattering and $\mu$ SR experiments", NIM A, 764 (2014) 156. https://doi.org/10.1016/j.nima.2014.07.029 